\title{
Penetration and Diffusion of X-Rays. Calculation of Spatial Distributions by Polynomial Expansion ${ }^{1}$
}

\author{
Lewis V. Spencer and U. Fano
}

\begin{abstract}
The expansions of the spatial distribution of X-rays in suitable systems of polynomials appear to converge rapidly. Calculations are performed for various source geometries in an infinite homogeneous medium. The surprisingly good convergence of this method of calculation is discussed with a view to its possible use in a variety of problems.
\end{abstract}

\section{Introduction}

Theoretical work at the National Bureau of Standards on the propagation of X-rays through large masses of homogeneous materials has so far been directed primarily to the study of extremely deep penetrations $[1,2,3] .^{2}$ In the complementary case of shallow penetration it was thought that a straightforward calculation of the effect of successive Compton scattering processes would prove convenient. The intention in this paper is to present a different method of calculation that can be carried out conveniently for moderate penetrations (up to 10 to 15 mean free paths of the primary radiation) and that relies on appropriate application of conventional physico-mathematical techniques.

The propagation of $\mathrm{X}$-rays through an infinite medium is governed by the transport equation

$$
\begin{aligned}
& \boldsymbol{u} \cdot \operatorname{grad} N(\boldsymbol{r}, \boldsymbol{u}, \lambda)=-\mu(\lambda) N+\int_{0}^{\lambda} d \lambda^{\prime} k\left(\lambda^{\prime}, \lambda\right) \\
& \int_{4 \pi} \boldsymbol{d} \boldsymbol{u}^{\prime} \frac{1}{2 \pi} \delta\left(1-\boldsymbol{u} \cdot \boldsymbol{u}^{\prime}-\lambda+\lambda^{\prime}\right) N\left(\boldsymbol{r}, \boldsymbol{u}^{\prime}, \lambda^{\prime}\right)+\text { source }
\end{aligned}
$$

where

$N(\boldsymbol{r}, \boldsymbol{u}, \lambda)$ is the density of photons of wavelength $\lambda$ (in Compton units), at the point $\boldsymbol{r}$ traveling in the direction $\boldsymbol{u}$ per unit solid angle; $\mu(\lambda)$ is the total narrow beam absorption coefficient; $k\left(\lambda^{\prime}, \lambda\right)$ is the Klein-Nishina differential coefficient for Compton scattering with a wavelength change from $\lambda^{\prime}$ to $\lambda$; and $\delta(x)$ is Dirac's delta-function.

It is widely known from the study of transport equations of type (1) that successive moments of the space distribution $N$ are related by a recurrence relationship. The zero-th moment, that is, the simple space integral of $N$, has already been calculated numerically in a number of sample cases $[4,5]$. A few more moments can be calculated numerically without excessive labor by manual operation. Automatic computers might prove useful for the same purpose.*

\footnotetext{
1 Work supported by the Office of Naval Research, Mathematics Branch. 2 Figures in brackets indicate the literature references at the end of this paper. * An extensive program of applications is now in progress under the sponsorship of the Atomic Energy Commission, New York Operations Office.
}

It is also widely recognized that a knowledge of the moments of a distribution function enables one in principle to reconstruct the function itself. However, little effort seems to have been applied along this line in the study of X-ray or neutron diffusion problems, presumably because the method was not expected to prove convenient. Actually, the moment method can be used very conveniently to obtain rapidly convergent expansions of the distribution function in suitable polynomial systems.

\section{General Discussion of Polynomial Expansions}

Consider, for simplicity, a distribution function $f$ of a single space variable $x$, which ranges from $-\infty$ to $+\infty$; $f$ is assumed to vanish sufficiently rapidly at both ends of this range. To obtain a polynomial expansion of $f$, a suitable weight function $w(x)$ is first chosen that vanishes at $\pm \infty$. One then chooses a succession of polynomials $p_{0}, p_{1}(x), \ldots p_{n}(x) \ldots$, which has an adjoint succession (also polynomials) $p_{0}^{+}, p_{1}^{+}(x)$. . ., etc., such that

$$
\int_{-\infty}^{\infty} w(x) p_{n^{\prime}}^{+}(x) p_{n}(x) d x=\delta_{n n^{\prime}} .
$$

One can then write

$$
f(x)=w(x) \Sigma_{n} a_{n} p_{n}(x) .
$$

The coefficients $a_{n}$ are given by the standard formula:

$$
a_{n}=\int_{-\infty}^{\infty} p_{n}^{+}(x) f(x) d x .
$$

(In connection with each function $w(x)$, one may, but need not, use the special set of polynomials $p_{n}$ which are identical with their adjoints $p_{n}^{+}$).

Knowledge of all the moments $\int_{-\infty}^{\infty} x^{m} f(x) d x$ of the distribution function determines the values of all the coefficients $a_{n}$ and thereby the complete expansion (3) with the preselected weight function and polynomials. If only the first $r$ moments are known, 
the first $r+1$ coefficients may be determined exactly, if the $(r+1)$-th adjoint polynomial is of degree $r$.

If the function $f(x)$ is the solution of a transport equation, it may not be necessary to calculate the moments first and thence the coefficients $a_{n}$. There exist recurrence relationships between the successive coefficients $a_{n}$, which are analogous to the relationships between the successive moments. One may then calculate the $a_{n}$ 's one after the other directly.

The significance of the expansion (3) of a distribution function into a series of polynomials is illustrated by the following argument: The first term of the series contains a polynomial of grade zero, that is, the constant $a_{0} p_{0}$. Therefore the weight function $w(x)$ itself represents the initial approximation to the function $f(x)$ to within the factor $a_{0} p_{0}$. If $w(x)$ were chosen just equal to the unknown function $f(x)$, the expansion would reduce to a single term. In general, one can expect the expansion to converge rapidly if he chooses a weight function that constitutes a sufficiently good approximation to $f(x)$.

For example, one may think of embodying into $w(x)$ advance information that may be available on the behavior of $f(x)$ for very large and very small values of $x$. It is a matter of convenience in any particular application how complicated one should make $w(x)$ to obtain a rapidly convergent expansion. In the practical application to the $\mathrm{X}$-ray problem, the choice of the simple exponential function for $w(x)$ yields convergence that seems quite adequate for a surprisingly large range of $x$.

The successive terms of the expansion may be described as corrective terms that are added to the initial approximation $w(x) \approx f(x)$ in order that successive moments of the approximation function

$$
w(x)\left[a_{0} p_{0}+a_{1} p_{1}(x)+a_{2} p_{2}(x)+\cdots \cdots\right],
$$

coincide with successive moments of the actual function $f(x)$. For example, suppose that an initial approximation $w(x) \approx f(x)$ has been chosen so that $\int_{-\infty}^{\infty} w(x) d x=\int_{-\infty}^{\infty} f(x) d x$ (i. e., $a_{0} p_{0}=1$ ). Then, it may turn out that the actual value of the first moment $\int_{-\infty}^{\infty} x f(x) d x$ is larger than the "estimated" value $\int_{-\infty}^{\infty} x w(x) d x$. To correct for this discrepancy the initial approximation should be modified by the addition of a corrective term $w(x) a_{1} p_{1}(x)$ which increases $w(x)$ on the side of positive $x$ and reduces it on the negative side. Actual calculation of high order moments should usually be required only if one has to fit the "tails" of the distribution function $f(x)$. It should not introduce substantial corrections to the main body of the distribution.

\section{Illustrative Examples}

As a demonstration, we have examined the Laguerre polynomial expansions of two otherwise known distribution functions, namely $\exp (-x)$ $J_{0}(2 i \sqrt{x})$ and $\exp (-x)_{1} F_{1}(-3 / 2,1,-x)$, for $x \geq 0$.
(Laguerre polynomials are the self-adjoint polynomials with the weight factor exp $(-x)$ over the range 0 to $\infty)$. These distributions represent the solutions of schematized X-ray or neutron problems $[1,6]$; the former pertains to the constant mean free path case $(\mu(\lambda)=$ const. $)$, the second to the case in which $\mu(\lambda)=a+b \lambda$.

The values obtained from successive polynomial approximations of the "build-up factors" $J_{0}(2 i \sqrt{x})$ and ${ }_{1} F_{1}(-3 / 2,1,-x)$ are compared with the exact values in tables 1 and 2 . The fit is surprisingly good. ${ }^{3}$ The addition of one more polynomial to the expansion appears to extend the range of satisfactory fit by at least an interval $\delta x \sim 3$ into the tail of the distribution function in the direction of increasing $x$. The tables include the results of expansions in Laguerre polynomials, as well as expansions in the related set of $U_{n}$ polynomials that are introduced in the next section.

TABLE 1. Representation of $J_{0}\left(2_{i} \sqrt{x}\right)$ by various finite sums of $L_{n}(x)$ and $U_{n}(x)$ polynomials

\begin{tabular}{|c|c|c|c|c|c|c|c|c|}
\hline \multirow{2}{*}{$x$} & \multicolumn{5}{|c|}{ Number of Laguerre polynomials } & \multirow{2}{*}{ Exact } & \multicolumn{2}{|c|}{$\begin{array}{l}\text { Number of } U_{n} \\
\text { polynomials }\end{array}$} \\
\hline & 3 & 4 & 5 & 6 & 7 & & 4 & 5 \\
\hline 0 & 1. 359 & 0. 9062 & 1. 020 & 0.9968 & 1.001 & 1 & 0.4797 & 0.9961 \\
\hline 1 & 2. 039 & 2. 341 & 2. 270 & 2. 281 & - & 2. 280 & 2.545 & 2. 280 \\
\hline 2 & 4. 077 & 4. 228 & 4. 265 & _...... & & 4. 251 & 4. 530 & 4. 289 \\
\hline 4 & 12. 23 & 11. 18 & 11. 29 & 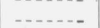 & & 11. 30 & 10.82 & 11. 28 \\
\hline 6 & 25.82 & 25.37 & 24.81 & & & 24.88 & 24.47 & 24.72 \\
\hline 8 & 44.85 & 50.67 & 49.57 & & & 49.21 & 50.60 & 49. 27 \\
\hline 10 & 69.32 & 90.01 & 91.25 & & $\ldots$ & 90.51 & 94.34 & 91.47 \\
\hline 12 & 99.22 & 146.8 & 157.8 & & & 157.7 & 160.8 & 159.8 \\
\hline 14 & -.... & 227.1 & 261.6 & 264.8 & & 264.0 & 255.2 & 264.4 \\
\hline 16 & -.... & 332.0 & 411.8 & 427.9 & & 427. 6 & 382.5 & 417.7 \\
\hline 18 & $\ldots$ & 465. 7 & 622.8 & 669.6 & 674.3 & 673.5 & 547.9 & 633.7 \\
\hline 20 & ..... & 632.2 & 910.3 & 1018 & 1038 & 1037 & -...... & 928.3 \\
\hline
\end{tabular}

TABLE 2. Representation of ${ }_{1} F_{1}(-3 / 2,1,-x)$ by a sum of four terms of the Laguerre or $U_{n}$ expansion

\begin{tabular}{|c|c|c|c|}
\hline$x$ & Laguerre & $U_{n}$ & Exact \\
\hline 0 & 0.9953 & 0.9798 & 1 \\
\hline 1 & 2. 681 & 2. 688 & 2. 678 \\
\hline 2 & 4. 677 & 4. 688 & 4. 680 \\
\hline 4 & 9.509 & 9.497 & 9. 512 \\
\hline 6 & 15. 31 & 15.21 & 15. 29 \\
\hline 8 & 21.90 & 21.92 & 21.89 \\
\hline 10 & 29. 13 & 29.30 & 29. 22 \\
\hline 12 & 36.84 & 37.23 & 37.21 \\
\hline 14 & 44. 72 & 45.65 & $45.8 \theta$ \\
\hline 16 & 52.73 & 54.42 & 54. 99 \\
\hline 18 & 60.66 & 63.41 & 64.71 \\
\hline 20 & 68.32 & 72.49 & 74.93 \\
\hline
\end{tabular}

To illustrate further the workings of these expansions, one may consider the integral representation of the distribution functions as "inverse Laplace transforms". In the case of the Bessel function, we have

${ }^{3}$ H. Hurwitz, Jr. has informed us that a similar surprisingly accurate fit was obtained in neutron calculations following a method of Placzek quoted in [7], p. 211 and following. 


$$
\begin{aligned}
e^{-x} J_{0}(2 i \sqrt{x}) & =e^{-x} \frac{1}{2 \pi i} \int_{\gamma-i \infty}^{\gamma+i \infty} e^{t x+\frac{1}{t}} \frac{d t}{t} \\
& =e^{-x+1} \frac{1}{2 \pi i} \int e^{t x+\frac{1-t}{t}} \frac{d t}{t} \\
& =e^{-x+1} \frac{1}{2 \pi i} \int e^{t x} \Sigma_{n} \frac{1}{n !}\left(\frac{1-t}{t}\right)^{n} \frac{d t}{t} \\
& =e^{-x+1} \Sigma_{n} \frac{1}{n !} L_{n}(x)
\end{aligned}
$$

where the $L_{n}$ 's are the normalized Laguerre polynomials. Thus, the expansion in Laguerre polynomials is obtained by expanding the Laplace transform of the distribution function into powers of $(1-t) / t$. This expansion converges rapidly near $t=1$; that is, in the same general region where the integrand exhibits a "saddle point" for $x \sim 1$. Each term of the expansion has a pole at the point $t=0$, where the Laplace transform is singular. An expansion including about four terms affords a good approximation of the Laplace transform from $t=\infty$ (saddle point for $x=0$ ) to perhaps $t=1 / 3$ (saddle point for $x=9$ ).

\section{Calculation of Polynomial Expansions}

The procedure for obtaining a convenient polynomial expansion of the distribution of X-ray photons varies somewhat depending upon the geometry of the source. Therefore, a number of different cases will be treated separately.

\section{Plane Monodirectional Source: High-Energy Photons Only}

The simplest case, perhaps, is that of a parallel beam of X-rays of infinite extension that enters a material perpendicularly through the plane $x=0$. The presence of the entrance surface does not cause any difficulty in this case because no high energy secondary X-rays will be present at the boundary.

In this case the photon density $N(\boldsymbol{r}, \boldsymbol{u}, \lambda)$ depends only on the components $x$ and $u_{x}$ of the vectors $\boldsymbol{r}$ and $\boldsymbol{u}$. The dependence of $N$ upon the direction cosine $u_{x}$ may be expressed by means of an expansion in Legendre polynomials

$$
N\left(x, u_{x}, \lambda\right) \sum_{l}=\frac{2 l+1}{4 \pi} N_{l}(x, \lambda) P_{l}\left(u_{x}\right) .
$$

The incident beam is represented in eq 1 by the source term

$$
\text { Source }=\delta(x) \frac{\delta\left(1-u_{x}\right)}{2 \pi} f(\lambda),
$$

where $f(\lambda)$ is the incident spectrum.

Multiplication of eq 1 by $P_{l}\left(u_{x}\right)$, followed by integration over $\boldsymbol{u}$ yields:

$$
\begin{array}{r}
\frac{1}{2 l+1}\left[(l+1) \frac{\partial N_{l+1}}{\partial x}+l \frac{\partial N_{l-1}}{\partial x}\right]=-\mu(\lambda) N_{l}(x, \lambda)+ \\
\int_{0}^{\lambda} d \lambda^{\prime} k\left(\lambda^{\prime}, \lambda\right) P_{l}\left(1-\lambda+\lambda^{\prime}\right) N_{l}\left(x, \lambda^{\prime}\right)+\delta(x) f(\lambda)
\end{array}
$$

The deep penetration of X-rays is controlled by the absorption of their most penetrating components.[1]. Therefore, one may describe the dependence of the photon density on the depth of penetration $x$ by means of a sum of polynomials in $x$ with the weight factor $\exp (-\alpha x)$. An obvious choice for the parameter $\alpha$ in the weight factor is the value of $\mu(\lambda)$ for the most penetrating component of the radiation, but a less obvious choice of $\alpha$ might well prove better.

As we mentioned earlier, the ortho-normal set of polynomials corresponding to the weight factor exp $(-\alpha x)$ is the set of Laguerre polynomials given by $L_{n}(\alpha x)=\Sigma_{v}\left(n ! /(n-v) ! v !^{2}\right)(-\alpha x)^{0}$. We accordingly represent the function $N_{l}(x, \lambda)$ in the form

$$
\begin{gathered}
N_{l}(x, \lambda)=e^{-\alpha x} \Sigma_{n} a_{l n}(\lambda) L_{n}(\alpha x), \\
a_{l n}(\lambda)=\int_{0}^{\infty} L_{n}(\alpha x) N_{l}(x, \lambda) \alpha d x .
\end{gathered}
$$

The equation that determines $a_{l n}(\lambda)$ is found by multiplying eq 8 by $\alpha L_{n}(\alpha x)$ and integrating over $x$ from 0 to $\infty$. The integration of the left side of (8) is carried out by parts. The values of $N_{l+1}$ and $N_{l-1}$ at the limits 0 and $\infty$ vanish as shown by the following arguments:

1. No radiation penetrates to $x=\infty$;

2. The density of incoming photons at the entrance surface should be taken equal to zero if the source term $\delta(x)$ is included in the range of integration;

3. There are no photons going back out of the entrance surface, as the treatment is limited to high photon energies.

The following identity is used in carrying out the remaining integration, which involves the derivative of the Laguerre polynomial

$$
\frac{d L_{n}(y)}{d y}=-\sum_{n^{\prime}=0}^{n-1} L_{n^{\prime}}(y)
$$

The integrals of all terms of (8) are thus reduced to the form (9a). The result is

$$
\begin{gathered}
\frac{\alpha}{2 l+1} \sum_{n^{\prime}=0}^{n-1}\left[(l+1) a_{l+1, n^{\prime}}+l a_{l-1, n^{\prime}}\right]=-\mu(\lambda) a_{l n}(\lambda)+ \\
\int_{0}^{\lambda} d \lambda^{\prime} k\left(\lambda^{\prime}, \lambda\right) P_{l}\left(1-\lambda+\lambda^{\prime}\right) a_{l n}\left(\lambda^{\prime}\right)+\alpha f(\lambda) .
\end{gathered}
$$

These equations coincide, for $n=0$, with those whose numerical solution has been discussed in a previous paper [5]. The equations for $n>0$ can be solved in succession by the same method, because 


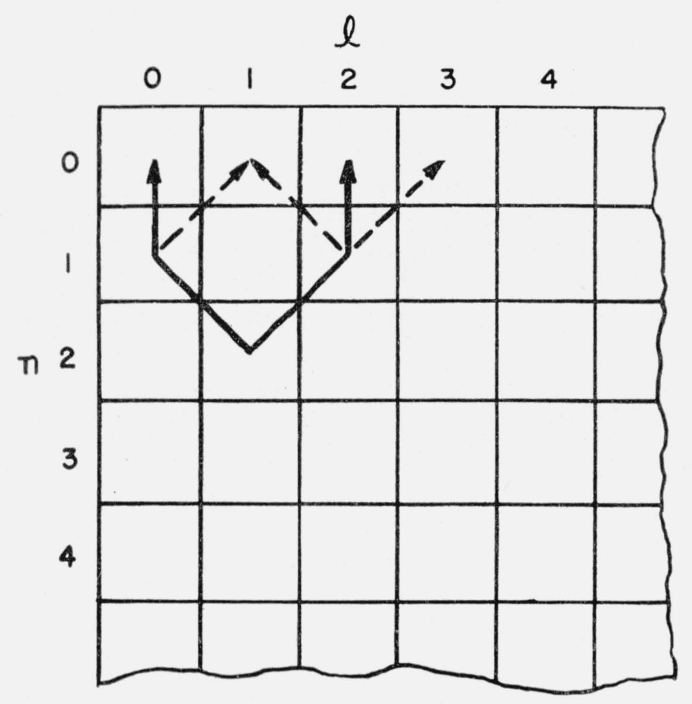

Figure 1. Chain linkages of the Legendre-Laguerre coefficients.

the sum on the left side contains only terms with $n^{\prime}<n$.

It is often of interest to know the density of photons at a point irrespective of their direction. This density is represented by the Legendre coefficient $N_{0}(x, \lambda)$. Calculation of the coefficients $a_{0 n}(\lambda)$ of the Laguerre expansion of $N_{0}$ requires, according to (10), the calculation of a set of coefficients $a_{l n}$ with $l>0$. Figure 1 shows diagrammatically how, for instance, the solution of the equation which determines $a_{12}(\lambda)$ requires a prior knowledge of the functions $a_{01}, a_{00}, a_{21}$, and $a_{20}$. In turn, the determination of $a_{01}$ and $a_{21}$ requires a prior knowledge of $a_{10}$ and $a_{30}$. If one calculates the moments

$$
b_{l n}=\int_{0}^{\infty} x^{n} N_{l}(x, \lambda) d x,
$$

instead of calculating directly the Laguerre coefficients $a_{l n}$, one finds the simpler scheme of interdependence illustrated by figure 2 . Here, solution of the equation for $b_{l n}$ requires prior knowledge of $b_{l-1, n-1}$ and $b_{l+1, n-1}$; thus, to calculate $a_{00}, a_{01}, a_{02}$, and $a_{03}$ one must also calculate $a_{10}, a_{20}, a_{30}, a_{11}, a_{21}$, and $a_{12}$; that is, one must solve numerically a total of 10 equations This does not involve an excessive amount of labor.

It may be interesting to relate the representation of the photon density by means of Laguerre polynomials to its representation as an inverse Laplace transform:

$$
N_{l}(x, \lambda)=\frac{1}{2 \pi i} \int e^{-p x} \phi_{l}(p, \lambda) d p .
$$

The moments of the distribution $N_{l}$ in depth correspond to the coefficients of the Taylor expansion of $\phi_{l}(p, \lambda)$ in powers of $p$ [7]. The expansion of $N_{l}$ in Laguerre polynomials is obtained by expanding $\phi_{l}$ into a series of terms of type $a_{n} p^{n} /(\alpha-p)^{n+1}$.

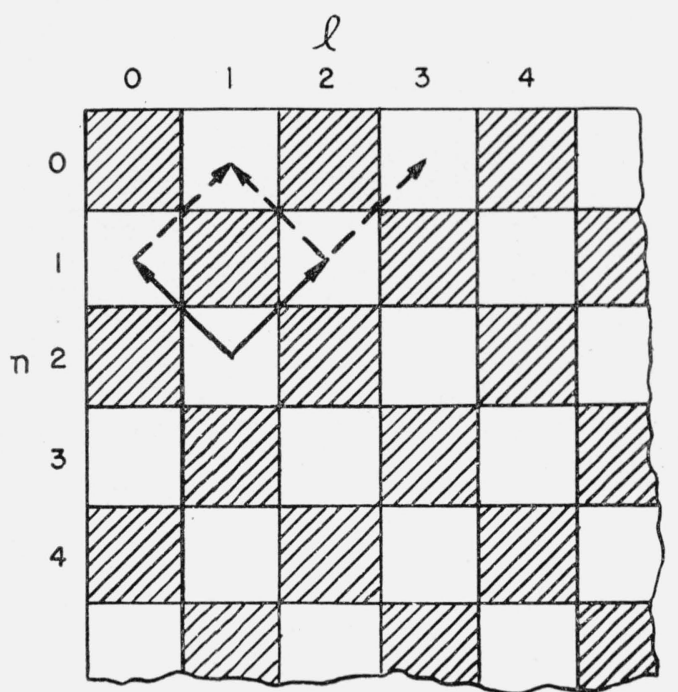

FIGURE 2. Chain linkages of the spatial moments of the Legendre coefficients.

Shaded and unshaded sets of boxes are not interlinked.

Each of these terms has a pole at $p=\alpha$. Notice that $\phi_{l}$ itself has a singularity at $p=\mu_{s}$, where $\mu_{s}$ is the smallest value of $\mu(\lambda)[2]$. Thus, the expansion of the Laplace transform in powers of $p /(\alpha-p)$ may be expected to converge more rapidly than the expansion in powers of $p$ if $\alpha$ is given a value near that of $\mu_{s}$.

\section{Plane Isotropic Source}

This case, in which radiation is emitted in all directions from all points of a plane, is familiar in the study of neutron penetration and diffusion $[6,7]$.

The distribution from a plane source, isotropic as well as monodirectional, depends only on the coordinate $x$ of each point $r$ and on the component $u_{x}$ of the direction of flow $\boldsymbol{u}$. The isotropic source term to be entered in eq 1 takes the form:

$$
\text { Source }=\frac{\delta(x) f(\lambda)}{4 \pi} .
$$

Correspondingly, one finds, instead of eq 8

$\frac{1}{2 l+1}\left[(l+1) \frac{\partial N_{l+1}}{\partial x}+l \frac{\partial N_{l-1}}{\partial x}\right]=-\mu(\lambda) N_{l}(x, \lambda)$
$+\int_{0}^{\lambda} d \lambda^{\prime} k\left(\lambda^{\prime}, \lambda\right) P_{l}\left(1-\lambda^{\prime}+\lambda\right) N_{l}\left(x, \lambda^{\prime}\right)+\delta(x) f(\lambda) \delta_{l 0}(12)$

If the source is surrounded by an infinitely extended medium, the radiation is distributed symmetrically with respect to the source plane $x=0$

$N\left(x, u_{x}, \lambda\right)=N\left(-x,-u_{x}, \lambda\right) ; \quad N_{l}(x, \lambda)=(-1)^{l} N_{l}(-x, \lambda)$. 
In this case the moments of the space distribution involve an integration over the entire range $-\infty<x<\infty$. Owing to the symmetry conditions (13), if $l$ is even, all the odd moments of $N_{l}$ are zero and if $l$ is odd, all the even moments of $N_{l}$ are zero.

In the preceding case the $n$th Laguerre polynomial added to the expansion reflected, in essence, information gathered from the calculation of the $n$-th moment of the distribution function. In the present case every other moment vanishes; that is, does not contribute to our knowledge of the distribution function; therefore, the method applied in the preceding case requires some modification. The $n$-th polynominal should now reflect information provided by the value of the $2 n$-th or $2 n+1$-th moment. An appropriate set of polynomials is required for this purpose.

The weight function should be selected in such a way that the distribution function has the correct symmetry. To maintain an analogy with the case of a monodirectional source, one may choose the weight function $\exp (-\alpha|x|)$ for the expansion of the even- $l$ Legendre coefficients $N_{l}(x, \lambda)$. Correspondingly, for odd values of $l$ the weight function $x \exp (-\alpha|x|)$ is perhaps the simplest choice. We write

$$
\left.\begin{array}{ll}
N_{l}(x, \lambda)=e^{-\alpha|x|} \sum_{n=0}^{\infty} a_{l n}(\lambda) U_{n}(\alpha|x|), & l \text { even } \\
N_{l}(x, \lambda)=\alpha x e^{-\alpha|x|} \sum_{n=0}^{\infty} a_{l n}(\lambda) V_{n}(\alpha|x|), & l \text { odd }
\end{array}\right\}
$$

where $U_{n}$ and $V_{n}$ are polynominals of degree $n$ still to be determined.

As indicated in section IV, 1 , the first $n$ terms of each expansion should embody all the information that is provided by the values of the first $n$ usable moments of $N_{l}(x, \lambda)$. In other words, the higher terms of the expansion (14) should not contribute to the lower usable moments. In the case of even $l$, this implies that the component $\exp (-\alpha|x|) U_{n}(\alpha|x|)$ of the function $N_{l}(x, \lambda)$ has vanishing low-order even moments

$$
\int_{-\infty}^{\infty} x^{2 n^{\prime}} e^{-\alpha|x|} U_{n}(\alpha|x|) d x=0 \quad \text { for } n^{\prime}<n .
$$

Similarly:

$\int_{-\infty}^{\infty} x^{2 n^{\prime}+1} \alpha x e^{-\alpha \mid x !} V_{n}(\alpha|x|) d x=0 \quad$ for $n^{\prime}<n$.

These conditions determine the sets $U_{n}$ and $V_{n}$ to within a normalization constant. We find the following expressions

$$
\left.\begin{array}{l}
U_{n}(y)=\frac{(-1)^{n}}{2^{n} n !}\left[\frac{\partial}{\partial y}+1\right]^{2 n} \sum_{j=0}^{n} \frac{(n+j) !}{j !(n-j) ! 2^{j}} y^{n-j} \\
V_{n}(y)=\frac{1}{2(n+1)}\left[\frac{\partial}{\partial y}+1\right]^{2} U_{n}(y)
\end{array}\right\}
$$

A number of these polynomials are given in table $3 .^{4}$

TABLE 3. The first four $U_{n}$ and $V_{n}$ polynomials

\begin{tabular}{|l|l|}
\hline$U_{0}=1$ & $V_{0}=\frac{1}{2}$ \\
$U_{1}=\frac{1}{2}(1-y)$ & $V_{1}=\frac{1}{8}(3-y)$ \\
$U_{2}=\frac{1}{8}\left(3-5 y+y^{2}\right)$ & $V_{2}=\frac{1}{48}\left(15-9 y+y^{2}\right)$ \\
$U_{3}=\frac{1}{48}\left(15-33 y+12 y^{2}-y^{3}\right)$ & $V_{3}=\frac{1}{384}\left(105-87 y+18 y^{2}-y^{3}\right)$ \\
\hline
\end{tabular}

To determine coefficients of the expansion (14) one may utilize the sets of adjoint polynomials $U_{n^{\prime}}^{+}$ and $V_{n^{+}}^{+}$, which fulfill the orthogonality condition $(2)$ together with $U_{n}$ and $V_{n}$, respectively. These polynomials are

$$
\left.\begin{array}{l}
U_{n^{\prime}}^{+}(y)=\sum_{\nu=0}^{n^{\prime}} \frac{1}{(2 \nu) !}\left(\begin{array}{c}
n^{\prime} \\
\nu
\end{array}\right)\left(-y^{2}\right)^{\nu} \\
V_{n^{\prime}}^{+}(y)=-\sum_{\nu=1}^{n^{\prime}+1} \frac{1}{(2 \nu-1) !}\left(\begin{array}{c}
n^{\prime}+1 \\
\nu
\end{array}\right) \frac{\left(-y^{2}\right)^{\nu}}{y}
\end{array}\right\}
$$

We multiply eq 12 by $\alpha U_{n}^{+}(\alpha x)$, if $l$ is even, or by $\alpha V_{n}^{+}(\alpha x)$, if $l$ is odd, and integrate over $x$ from $-\infty$ to $\infty$. The integration proceeds as the integration of (8). The left side integrated by parts yields one term that vanishes and an integral that contains the derivative $d U_{n}^{+} / d x$ ( $l$ even) or $d V_{n}^{+} / d x$ ( $l$ odd). These derivatives fulfill the identities

$$
\left.\begin{array}{l}
\frac{d U_{n}^{+}(y)}{d y}=-V_{n-1}^{+}(y) \\
\frac{d V_{n}^{+}(y)}{d y}=\sum_{n^{\prime}=0}^{n} U_{n^{\prime}}^{+}(y)
\end{array}\right\}
$$

Thereby, the integration of eq 12 reduces to integrals of the type

$$
\left.\begin{array}{rl}
a_{l n}(\lambda) & =\int_{-\infty}^{\infty} U_{n}^{+}(\alpha x) N_{l}(x, \lambda) d x \\
& =\sum_{\nu=0}^{n}\left(\begin{array}{c}
n \\
\nu
\end{array}\right) \frac{(-1)^{\nu}}{(2 \nu) !} b_{l,,_{\nu}}(\lambda), \quad l \text { even } \\
a_{l n}(\lambda) & =\int_{-\infty}^{\infty} V_{n}^{+}(\alpha x) N_{l}(x, \lambda) d x \\
& =\sum_{\nu=1}^{n+1}\left(\begin{array}{c}
n+1 \\
\nu
\end{array}\right) \frac{(-1)^{\nu-1}}{(2 \nu-1) !} b_{l, 2 \nu-1}(\lambda), \quad l \text { odd }
\end{array}\right\}
$$

${ }^{4}$ It might be worth mentioning in passing that $U_{n}$ and $V_{n}$ are solutions of the following $3 \mathrm{~d}$ order differential equation, with the parameter $m$ equal to zero and one, respectively

$$
y \frac{d^{3} W}{d y^{3}}+(2 m+1-3 y) \frac{d^{2} W}{d y^{2}}+2(y-2 m-1) \frac{d W}{d y}-2 n W=0
$$

Higher associated polynomials exist having weight functions and adjoint polynomials analogous to those of $U_{n}$ and $V_{n}$. 


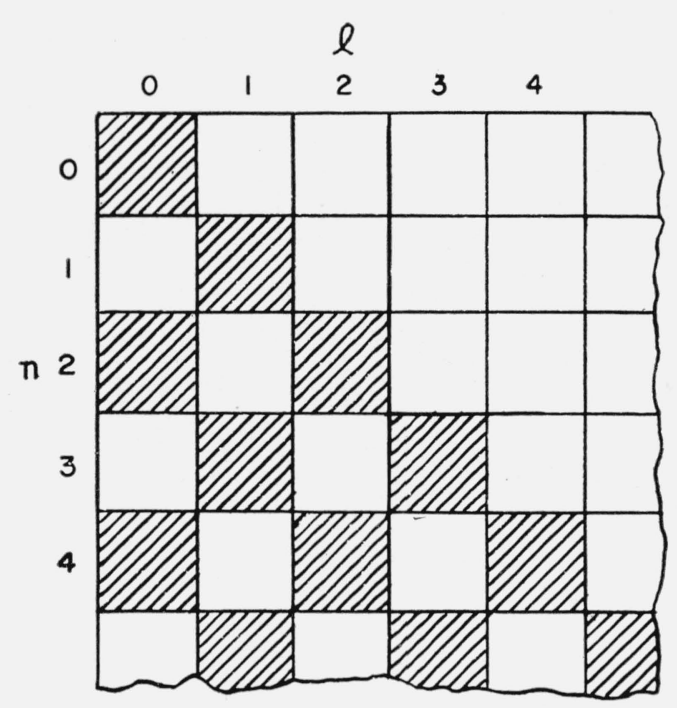

FIGURE 3.-Interlinkages for isotropic sources.

Shaded elements only are involved in the calculation.

where $b_{l, n^{\prime}}$ is again the $n^{\prime}$-th spatial moment of $N_{l}(x, \lambda)$. The result is

$$
\left.\begin{array}{c}
\frac{\alpha}{2 l+1}\left[(l+1) a_{l+1, n-1}+l a_{l-1, n-1}\right]=-\mu(\lambda) a_{l n}(\lambda)+ \\
\int_{\lambda_{0}}^{\lambda} d x^{\prime} k\left(\lambda^{\prime}, \lambda\right) P_{l}\left(1-\lambda+\lambda^{\prime}\right) a_{l n}\left(\lambda^{\prime}\right)+\alpha f(\lambda) \delta_{l 0}, \quad l \text { even } \\
\frac{\alpha}{2 l+1} \sum_{n^{\prime}=0}^{n}\left[(l+1) a_{l+1, n^{\prime}}+l a_{l-1, n^{\prime}}\right]=-\mu(\lambda) a_{l n}(\lambda)+ \\
\int_{\lambda_{0}}^{\lambda} d \lambda^{\prime} k\left(\lambda^{\prime}, \lambda\right) P_{l}\left(1-\lambda+\lambda^{\prime}\right) a_{l n}\left(\lambda^{\prime}\right), \quad \text { lodd }
\end{array}\right\}
$$

These equations can be solved chainwise, much like the eq 10. Notice that $a_{l n}=0$ for $n \leq(l / 2-1)$, since the source term is confined to the equation with $l=0$. The equations must be solved in the following order: $a_{00}, a_{10}, a_{21}, a_{31}, \cdots, a_{01}, a_{11}, a_{21} \cdots$, $a_{02}, a_{12}, \cdots, a_{03} \cdots$ Here again, as in the case of the monodirectional source, the calculation of four terms of the expansion of $N_{0}(x, \lambda)$ requires the solution of 10 equations.

The fact that $a_{l n}=0$ for $n \leq(l / 2-1)$ relates to the known fact that the moment $b_{n l}=\int_{-\infty}^{\infty} x^{n} N_{l}(x, \lambda) d x$ vanishes when $n<l[7]$. (Only the moments corresponding to the shaded boxes in fig. 3 are not zero. The value of each moment $b_{l n}$ depends on the values of the moments $b_{l+1, n-1}$, and $b_{l-1, n-1}$, as shown in fig. 2. The calculation must begin with $b_{00}$, whose equation is inhomogeneous, that is from the upper left corner of the scheme.) Accordingly, the Laplace transform of $N_{l}(x, \lambda)$, namely, $\phi_{l}(p, \lambda)$, has a Taylor expansion that begins with the $l$-th term. The polynomial expansions (14) of $N_{l}$ are obtained as inverse Laplace transforms if $\phi$ is represented as a series of terms $p^{2 n} /\left(\alpha^{2}-p^{2}\right)^{n+1}$, for even $l$, or $p^{2 n+1} /\left(\alpha^{2}-p^{2}\right)^{n+1}$ for odd $l$.

\section{Plane Monodirectional Source-No Energy Limitation}

The method developed for the treatment of X-rays from a plane isotropic source can be applied to the study of radiation from other plane sources. To this end, one must generalize the preceding treatment somewhat.

It will be remembered that because the source is symmetric and because of the interlocking of moments illustrated in figure 2 , the only useful moments are those which correspond to the black squares of the checkerboard pattern of figure 2. The halfcheckerboard pattern of useful moments (fig. 3) results from the isotropy of the source (eq 11). In the general symmetric case, source terms, will be present in all equations, not just those alone in which $l=0$; and the full checkerboard scheme of moments (fig. 2) will be used.

On the other hand, if the source were antisymmetric in $x$, the moments corresponding to the shaded squares in figure 2 would all be zero. The useful moments would be interrelated among themselves just as before and would correspond to the white squares of figure 2 .

One can take advantage of this separate interlocking of different sets of moments by regarding a plane monodirectional source as the superposition of a symmetrical and an antisymmetrical source. The symmetrical source component injects equal photon densities in opposite directions perpendicular to the plane $x=0$ on either side. The antisymmetrical source injects a photon density equal to that of the symmetrical source of the positive side of $x=0$ and a "negative" photon density of equal size on the negative side.

The photon distribution $N^{(a)}\left(x, u_{x}, \lambda\right)$ due to the antisymmetric source has symmetry properties opposite to (14), namely,

$$
\begin{aligned}
N^{(a)}\left(x, u_{x}, \lambda\right) & =-N^{(a)}\left(-x,-u_{x}, \lambda\right) ; \\
N_{l}^{(a)}(x, \lambda) & =(-1)^{l+1} N_{l}^{(a)}(-x, \lambda) .
\end{aligned}
$$

The polynomial expansions of the Legendre coefficients $N_{l}^{(a)}(x, \lambda)$ involve the $V_{n}$ polynomials for even $l$ and the $U_{n}$ polynomials for odd $l$, that is, they conform to (14) with opposite even-odd condition.

The total photon density from a plane monodirectional source is thus given by the sum of two terms

$$
N\left(x, u_{x}, \lambda\right)=N^{(s)}\left(x, u_{x}, \lambda\right)+N^{(a)}\left(x, u_{x}, \lambda\right) .
$$

$N^{(s)}$ is determined by a set of equations identical with (20), except that the source term does not include the factor $\delta_{l 0} . \quad N^{(a)}$ is determined by a similar system with even and odd $l$ interchanged.

The total density $N\left(x, u_{x}, \lambda\right)$ all but cancels on the negative side of the source plane, inasmuch as 
$N^{(a)}$ is negative there. The residual photon density for $x<0$ represents radiation that has been backscattered behind the source. It should consist of comparatively low energy photons.

\section{Point Isotropic Source}

The photon density generated by a point isotropic source can be derived from the solution corresponding to a plane isotropic source (case (b)) [7]. This derivation is especially simple if one calculates only the total density of photons at each point irrespective of their direction. For this reason we shall outline here only sketchily an independent calculation for the case of a point source.

Owing to the spherical symmetry of the problem, the distribution function $N(\boldsymbol{r}, \boldsymbol{u}, \lambda)$ depends only on the magnitude $r$ of $\boldsymbol{r}$ and on the component $u_{r}=\boldsymbol{u} \cdot \boldsymbol{r} / r$ of $\boldsymbol{u}$, if the origin of the coordinates is laid at the source. The source term in eq 1 takes the form $\delta(r) f(\lambda) / 4 \pi r^{2}$ and the transport eq 1 becomes

$$
\begin{aligned}
\boldsymbol{u} \cdot \operatorname{grad} N\left(r, u_{r}, \lambda\right)= & u_{r} \frac{\partial N}{\partial r}+\frac{\left(1-u_{r}^{2}\right)}{r} \frac{\partial N}{\partial u_{r}} \\
= & -\mu(\lambda) N+\int_{0}^{\lambda} d \lambda^{\prime} k\left(\lambda^{\prime}, \lambda\right) \\
& \int_{4 \pi} d \boldsymbol{u}^{\prime} \frac{1}{2 \pi} \delta\left(1-\boldsymbol{u} \cdot \boldsymbol{u}^{\prime}-\lambda+\lambda^{\prime}\right) \\
& N\left(r, u_{r}^{\prime}, \lambda^{\prime}\right)+\frac{\delta(r) f(\lambda)}{4 \pi r^{2}}
\end{aligned}
$$

The density $N$ can be expanded in Legendre polynomials of $u_{r}$, as in eq 6 . The transport equation takes then a form corresponding to (8), whose derivation makes use of the identity

$$
\begin{gathered}
\left(1-u_{r}^{2}\right) d P_{l} / d u_{r}=l(l+1)\left(P_{l-1}-P_{l+1}\right) /(2 l+1) . \\
\frac{(l+1)}{2 l+1}\left(\frac{l+2}{r}+\frac{\partial}{\partial r}\right) N_{l+1}(r, \lambda)-\frac{l}{2 l+1}\left(\frac{l}{r}-\frac{\partial}{\partial r}\right) N_{l-1}(r, \lambda) \\
=-\mu(\lambda) N_{l}(r, \lambda)+\int_{0}^{\lambda} d \lambda^{\prime} k\left(\lambda^{\prime}, \lambda\right) P_{l}\left(1-\lambda+\lambda^{\prime}\right) N_{l}\left(r, \lambda^{\prime}\right) \\
\quad+\frac{\delta(r) f(\lambda) \delta_{l 0}}{4 \pi r^{2}}
\end{gathered}
$$

In this case we do not derive directly the coefficients of an expansion of $N_{l}(r, \lambda)$ in polynomials in $r$, but we calculate the moments

$$
\int_{0}^{\infty} r^{n} N_{l}(r, \lambda) 4 \pi r^{2} d r=b_{l n}(\lambda) .
$$

To this end one multiplies (24) by $r^{n}$ and integrates over all the space. The result is

$$
\begin{aligned}
& \frac{1}{2 l+1}\left[(l+1)(l-n) b_{l+1, n-1}(\lambda)-l(l+n+1) b_{l-1, n-1}(\lambda)\right]= \\
& \quad-\mu(\lambda) b_{l n}(\lambda)+\int_{0}^{\lambda} d \lambda^{\prime} k\left(\lambda^{\prime}, \lambda\right) P_{l}\left(1-\lambda+\lambda^{\prime}\right) b_{l n}\left(\lambda^{\prime}\right) \\
& \quad+f(\lambda) \delta_{l 0} \delta_{n 0}
\end{aligned}
$$

This set of equations can be solved chainwise in exactly the same way as in the plane isotropic case. Coefficients of the $U_{n}$ and $V_{n}$ polynomials can be obtained by adding and subtracting moments. The coefficients in the expansion of the total density of photons in $U_{n}$ polynomials are related in the following way with the corresponding coefficients in the plane isotropic source case

$$
\left(a_{0 n}\right)_{\text {point }}=(2 n+1)\left(a_{0 n}\right)_{\text {plane }}-2 n\left(a_{0, n-1}\right)_{\text {plane }} .
$$

The coefficients $a_{l n}$ for the two sources in the case $l \neq 0$ are related in a more complicated way.

\section{Point Monodirectional Source}

This kind of source may properly be regarded as the elementary source, since any source can be represented as an aggregate of point monodirectional sources. The case of a point monodirectional source is more complicated than the cases considered above, because it involves explicitly the diffusion of radiation sidewise from the initial "line of fire."

Cylindrical coordinates $r=(z, \rho, \phi)$, with the $z$ axis lying along the line of fire are appropriate to the geometry of this problem. The direction vector $\boldsymbol{u}$ can be described by polar coordinates $(\theta, \Phi+\phi)$, so that the zero azimuth $\Phi=0$ refers to a plane through the $z$ axis and the point $\boldsymbol{r}$ under consideration. Owing to the cylindrical symmetry of the source the photon density depends explicitly on the variables in the following way

$$
N(\boldsymbol{r}, \boldsymbol{u}, \lambda)=N(z, \rho, \theta, \Phi, \lambda) .
$$

To calculate the expression $\boldsymbol{u} \cdot \operatorname{grad} N$ explicitly, one must consider that the gradient is taken for constant $\boldsymbol{u}$, which means $\delta \Phi=-\delta \phi$. Accordingly

$$
\begin{aligned}
& \boldsymbol{u} \cdot \boldsymbol{g r a d} N=\cos \theta \frac{\partial N}{\partial z}+\sin \theta \cos \Phi \frac{\partial N}{\partial \rho} \\
& -\frac{\sin \theta \sin \Phi}{\rho} \frac{\partial N}{\partial \Phi} .
\end{aligned}
$$

In this problem the distribution function should be expanded into Laplace spherical harmonics $Y_{l m}(\theta, \Phi)$ and moments should be taken with respect to both space coordinates $z$ and $\rho$. Therefore we introduce the set of dependent variables

$$
\begin{aligned}
N_{l m}^{n p}(\lambda)= & \int_{0}^{\infty} \rho^{p+1} d \rho \int_{-\infty}^{\infty} z^{n} d z \int_{0}^{\pi} \sin \theta d \theta \\
& \int_{0}^{2 \pi} d \Phi Y_{l m}^{*}(\theta, \Phi) N(z, \rho, \theta, \Phi, \lambda)
\end{aligned}
$$

A corresponding four-fold integration applied to eq 1 reduces this equation to the form 


$$
\begin{aligned}
& -\frac{1}{\sqrt{4(l+1)^{2}-1}}\left\{n \sqrt{(l+1)^{2}-m^{2}} N_{l+1, m}^{n-1, p}\right. \\
& -\frac{p+m}{2} \sqrt{(l-m+1)(l-m+2)} N_{l+1, m-1}^{n, p-1} \\
& \left.+\frac{p-m}{2} \sqrt{(l+m+1)(l+m+2)} N_{l+1, m+1}^{n, p-1}\right\} \\
& -\frac{1}{\sqrt{4 l^{2}-1}}\left\{n \sqrt{l^{2}-m^{2}} N_{l-1, m}^{n-1, p}\right. \\
& +\frac{p+m}{2} \sqrt{(l+m)(l+m-1)} N_{l-1, m-1}^{n, p-1} \\
& \left.-\frac{p-m}{2} \sqrt{(l-m)(l-m-1)} N_{l-1, m+1}^{n, p-1}\right\} \\
& =-\mu(\lambda) N_{l, m}^{n, p}(\lambda)+\int_{0}^{\lambda} d \lambda^{\prime} \mathrm{k}\left(\lambda^{\prime}, \lambda\right) P_{l}\left(1-\lambda+\lambda^{\prime}\right) N_{l, m}^{n, p}\left(\lambda^{\prime}\right) \\
& +f(\lambda) \delta_{n 0} \delta_{p 0} \delta_{m 0} \sqrt{\frac{2 l+1}{4 \pi}}
\end{aligned}
$$

This equation reduces to the equation for the plane monodirectional source, as it should do, when $p=m=0$. The coefficients $N_{l, 0}^{n, 0}(\lambda)$ can be calculated accordingly. Once this is done one can proceed to $p=1, m= \pm 1$, and then on again.

In the preceding cases the calculation of a threevariable function such as $N\left(r, w_{r}, \lambda\right)$ was reduced to the calculation of a two-dimensional array of coefficients $N_{l}^{n}(\lambda)$. The present problem involves five independent variables and reduces to the solution of an "array of problems," each of them as complicated as any of the previous ones and corresponding to one pair of values of $p$ and $m$. The genetic connections in the array of problems are represented in figure 4.

Various polynomial representations of the radial distribution might suggest themselves in the course of any practical application. The use of a gaussian weight function should presumably be convenient at least in the lower energy range.

\section{Numerical Applications*}

\section{Penetration of 10-Mev Gamma Rays in Lead}

The method of section IV, 1 was applied numerically to the case of a parallel beam of $10.2-\mathrm{Mev}$ photons entering an infinitely thick layer of lead. A heavy element was chosen because it is a more difficult situation to treat with asymptotic methods, owing to the minimum of the absorption coefficient near 3 Mev [2]. The same initial energy was chosen which is used by Karr and Hurwitz in their asymptotic calculation with the "straight ahead" approximation [8]. The calculation was extended only down to photon energies of about $1 \mathrm{Mev}$ $(=0.49)$, partly because of the limitation to the validity of the method indicated in section $I V, 1$.

*Additional applications were reported by L. V. Spencer and F. A. Stinson at the New York Meeting of the American Physical Society, February 3, 1951.
This calculation was carried out not onıy by the method of section IV, 1 but also with a corresponding "straight ahead" approximation in order to estimate the error involved in neglecting angular effects. The straight ahead approximation implies disregarding the effects of deflections experienced by high-energy photons in the course of Compton scattering. In this approximation one solves only the eq 8 for $l=0$, replacing $\partial N_{1} / \partial x$ by $\partial N_{0} / \partial x$, so that $N_{0}(x, \lambda)$ is the only distribution function to be determined.

Finally, the straight ahead calculation was carried out once more, neglecting the $\sin ^{2} \theta$ terms in the Compton cross section, that is, omitting the last two terms of the expression

$k\left(\lambda^{\prime}, \lambda\right)=\frac{3}{8}\left(\frac{\lambda^{\prime}}{\lambda}\right)^{2}\left[\frac{\lambda^{\prime}}{\lambda}+\frac{\lambda}{\lambda^{\prime}}-2\left(\lambda-\lambda^{\prime}\right)+\left(\lambda-\lambda^{\prime}\right)^{2}\right] \mu_{\text {Thomson }}$

The purpose of this calculation was partly to estimate the importance of these terms and partly to obtain a proper comparison with the work of Karr and Hurwitz, who used this approximation in making their calculation.

Four terms of the polynomial representation (9) of the function $N_{0}(x, \lambda)$ were worked out with $\alpha$ set equal to $\mu_{m}$, the minimum value of $\mu(\lambda)$. For comparison, the coefficients were regrouped to correspond to a polynomial expansion with $\alpha$ set equal to $\mu(\lambda=0.05)$ and also to a polynomial expansion with the weight factor $x \exp \left(-\mu_{m} x\right)$, the last being perhaps the most realistic weight factor in view of what is known about the initial and asymptotic behavior of the build-up factor. The results using these different weight factors were all in satisfactory agreement.

Figure 5 shows the differential spectrum given by calculation according to section IV, 1 . The energy density is plotted as a function of the energy for various penetrations. Notice the accumulation of energy in the range $\sim 3 \mathrm{Mev}$, corresponding to the minimum absorption coefficient.

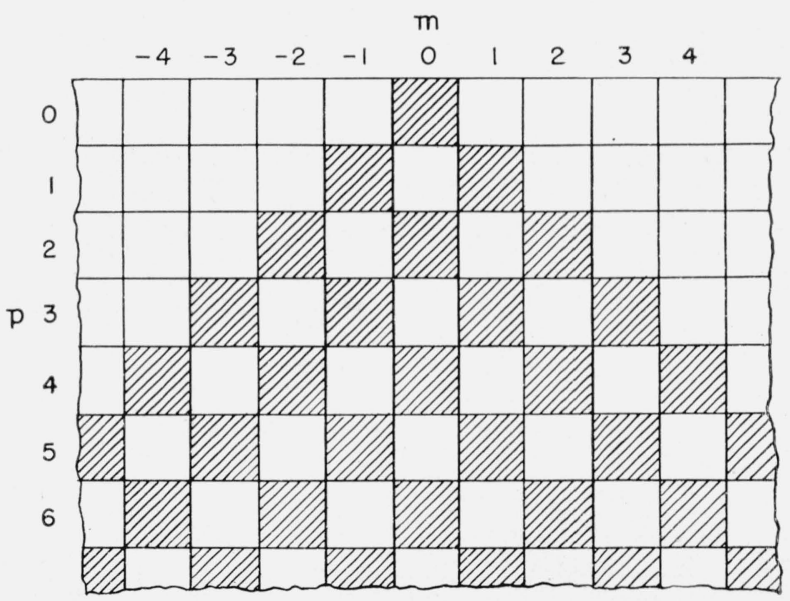

FIGURE 4. Interlinkages among the radial moments and the Fourier coefficients of the azimuthal distribution for a point monodirectional source.

Only shaded elements are involved in the calculation. 


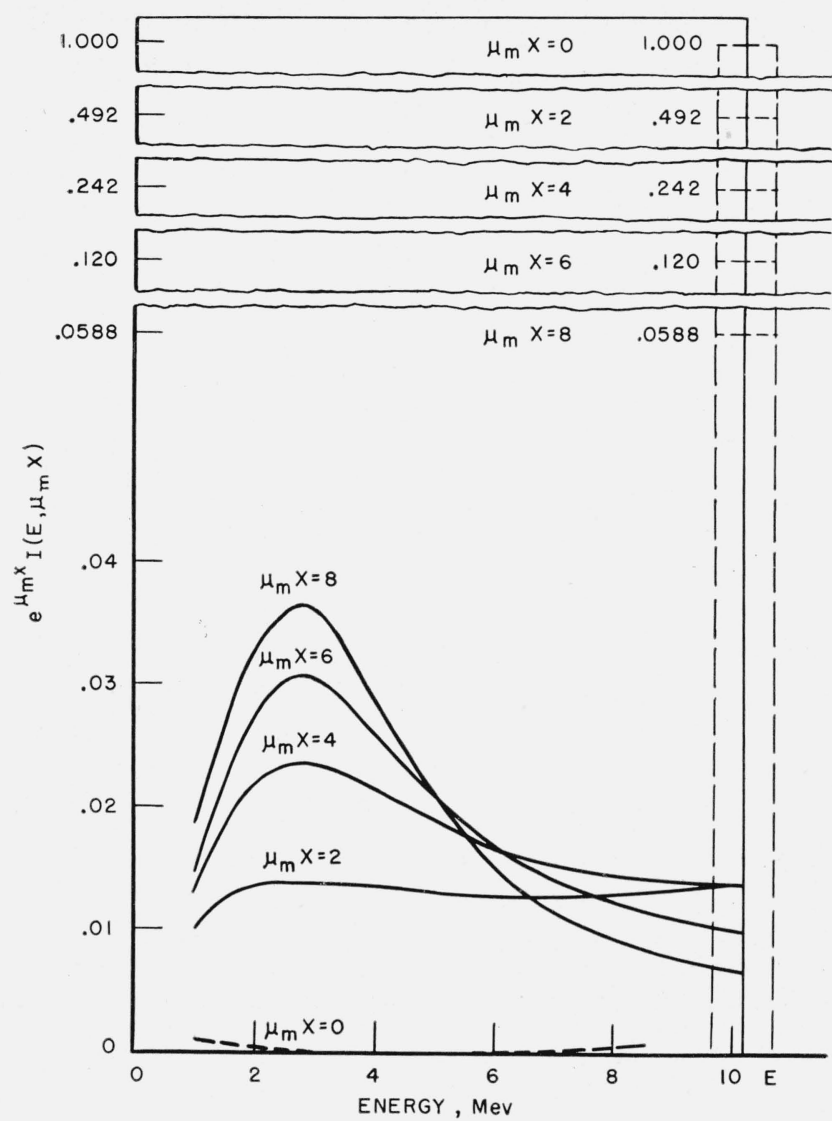

FiguRe 5. Differential spectra $I\left(E, \mu_{m} x\right)$ of the X-ray intensity at various distances from a plane monodirectional 10.2-Mev source in $\mathrm{Pb}$.

The position of the source spectrum is indicated by the solid vertical line, its intensity by the area of the dotted rectangle. The scale of ordinates is normalizec to unit strength of the source at $x=0$; at greater depths it discounts an exponential decay corresponding to the absorption coefficient $\mu(3.2 \mathrm{Mev})=\mu_{m}=0.469 \mathrm{~cm}^{-1}$ of the most penetrating component. The dotted line for $x=0$ departs from the base line because only a finite number of terms in the expansion were calculated.

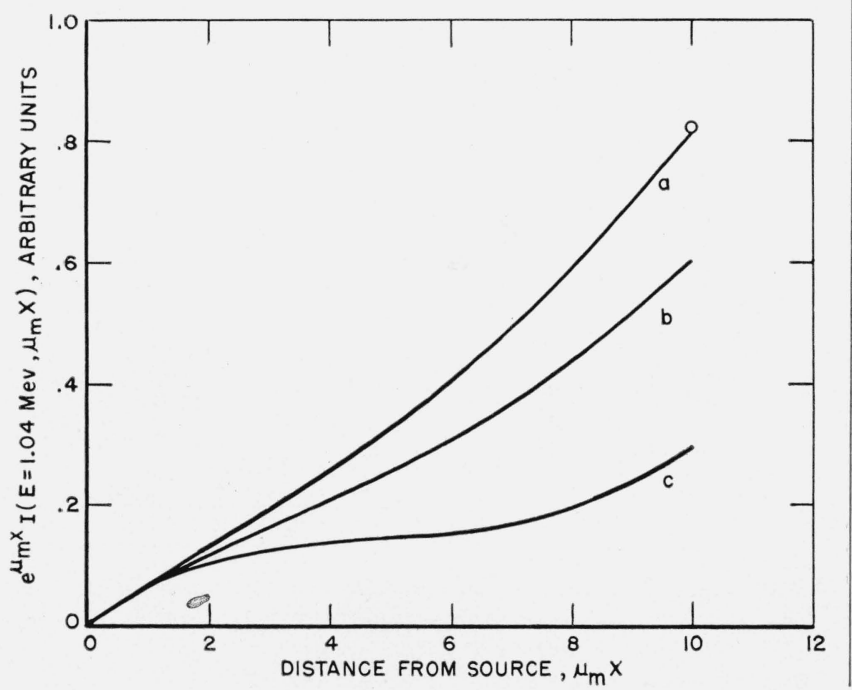

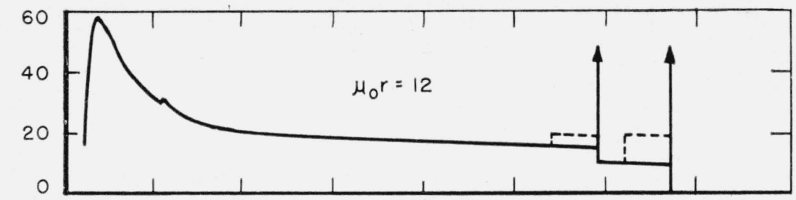
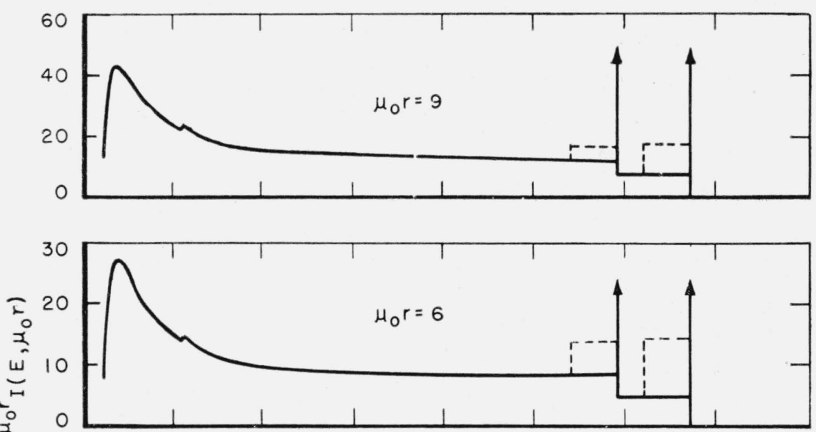

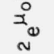
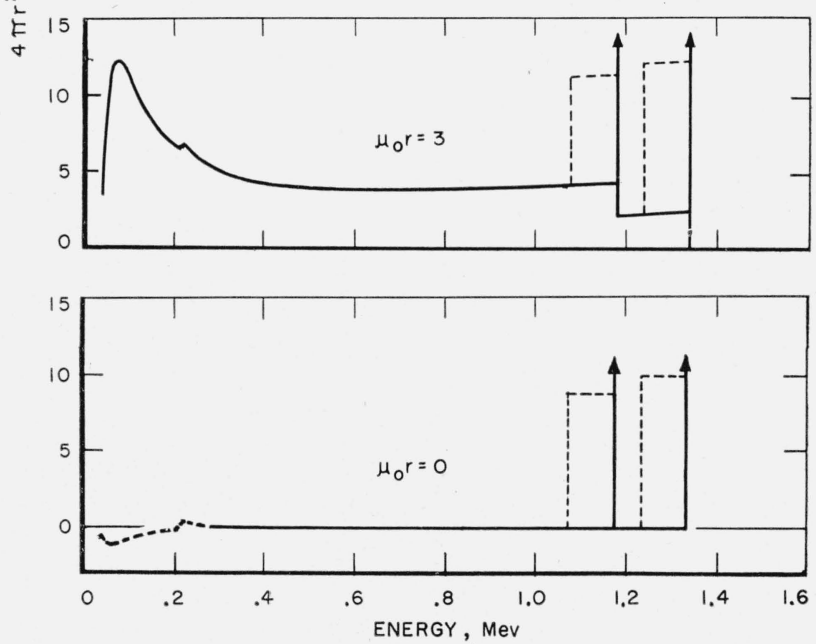

Figure 7. Differential spectra $I\left(E, \mu_{0} r\right)$ of the X-ray intensity at various distances $r$ from a $\mathrm{Co}^{60}$ point isotropic source in water.

The positions of the two lines of the source spectrum are indicated by the solid vertical lines, their intensities by the area of the dotted rectangles. The scales of ordinates are normalized to unit intensity of the $1.33-\mathrm{Mev}$ component of the source; $\mu_{0}=0.0612 \mathrm{~cm}^{-1}$ is the narrow-beam absorption coefficient of this component.

Figure 6. Differential intensity $I\left(E, \mu_{m} x\right)$ of the 1.04-Mev component of the spectrum of figure 5 as a function of the distance from the source, calculated by different methods: (a) as in figure 5 ("exact"), (b) disregarding the deflection of scattered photons ("straight ahead"), (c) using the extreme relativistic form of the Klein-Nishina cross section (also "straight ahead").

The point at the end of the top curve is the result of the Karr-Hurwitz asymptotic calculation. 


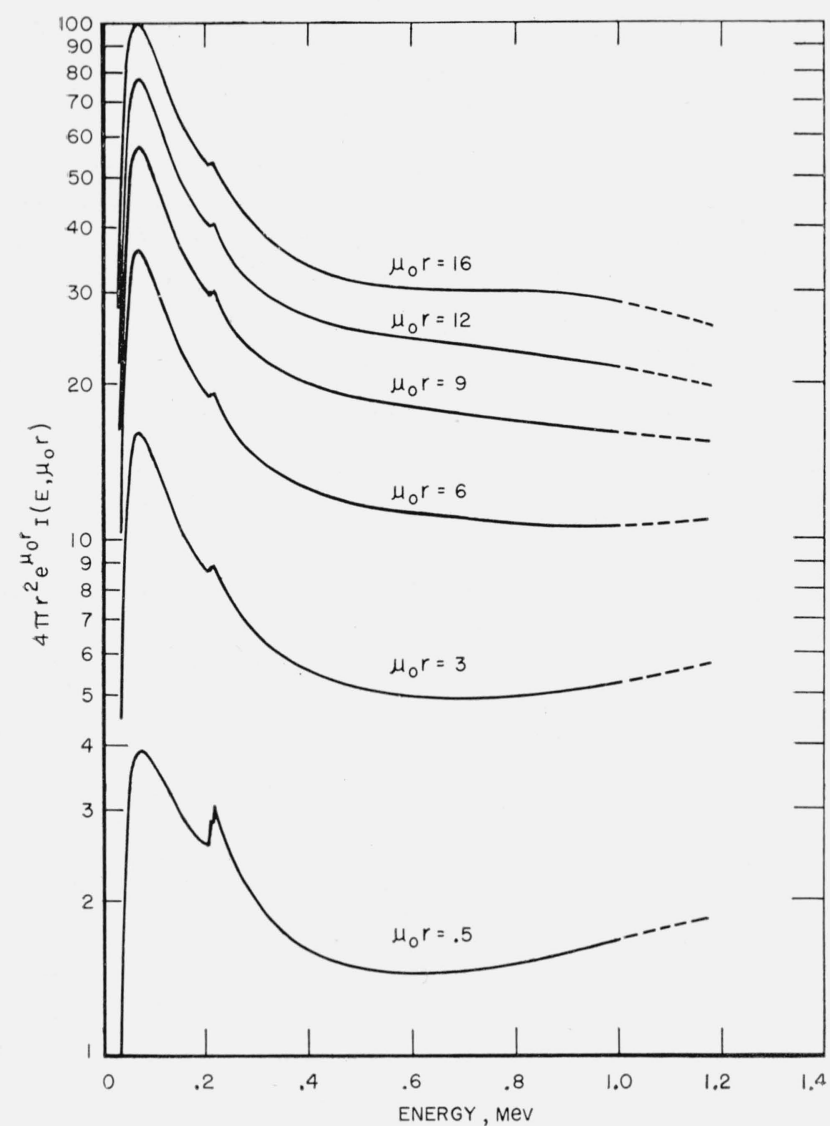

FIgure 8. Semilogarithmic plot of the spectra of figure 7 , which illustrates the trend toward equilibrium.

The higher energy portions of the spectra have been omitted for clarity.

Figure 6 shows the angular calculation compared with the two straight ahead calculations. The energy density of the component $\lambda=0.49$ is plotted against depth of penetration. Karr and Hurwitz carried their asympotitic calculations down to $\mu_{m} x=10$. It is possible to compare their results with ours at that penetration. The circle gives their value and is to be compared with our upper curve. The two values are much closer together than was expected from the accuracy of the calculations. Notice that the angular calculation differs by a factor of almost 3 from the Karr-Hurwitz calculation at 10 mean free paths of the most penetrating component.

\section{2. $\mathrm{Co}^{60}$ Isotropic Point Source in Water}

This problem has been investigated experimentally by White [9]. We have made numerical calculations by using the method outlined in section IV, 4 . The coefficients of the first $4 U_{n}$ polynomials in the expansion of $N_{0}(r, \lambda)$ have been evaluated. (Below $0.150 \mathrm{Mev}$, the coefficients of $U_{2}$ and $U_{3}$ were estimated from the trend of the lower coefficients, since approximate equilibrium was attained.)

Some results are illustrated in figures 7,8 , and 9 . Figure 7 shows the differential energy spectrum at

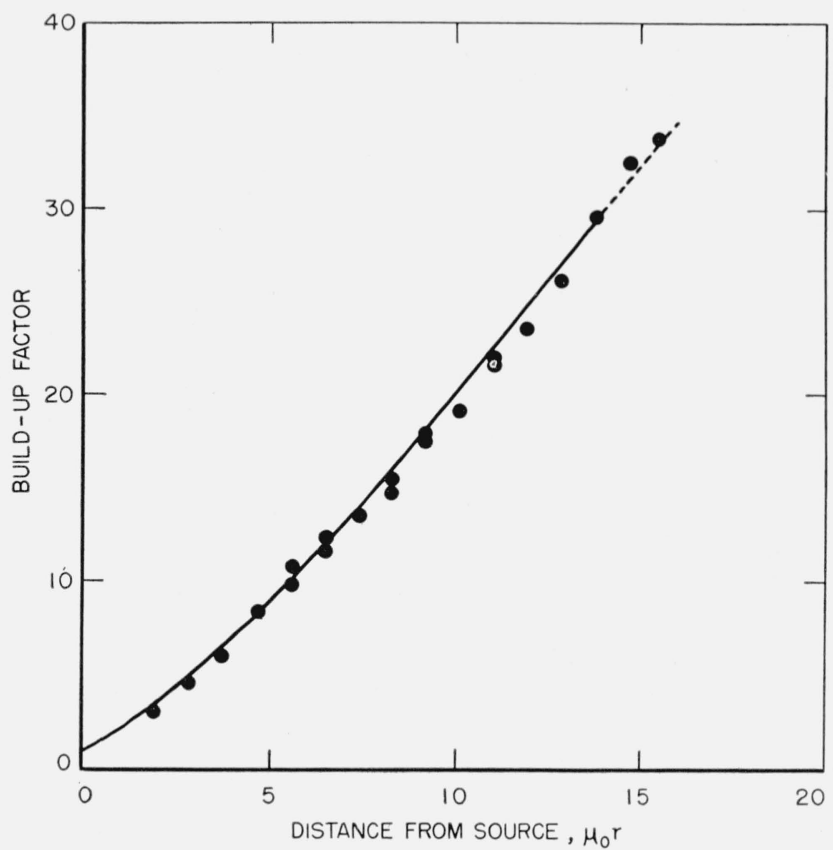

FIGURE 9. Comparison of the calculated curve for the build-up factor with the experimental results of [9].

various depths of penetration. The same results are plotted semilogarithmically in figure 8 to illustrate the approach to an equilibrium spectrum at the lower energies. At equilibrium the curves become parallel, indicating that the spectrum no longer changes with increasing penetration.

Figure 9 compares White's experimental buildup factors, as measured by an ionization chamber (and a counter), with a theoretical build-up factor. The latter was evaluated by weighting the calculated spectra with the theoretical response in roentgen of a standard free-air ionization chamber and then again with the specific correction factor of the Keleket III pocket dosimeter [10]. The integral response over all spectral components was divided by the calculated response to the primary radiation to give the theoretical build-up factor. The slight downward curvature at the end of the theoretical curve is an artifact that indicates that the 4-term approximation is beginning to fail at this distance.

\section{Discussion}

The analytical developments and the numerical applications presented in this paper seem to give some confidence in the following conclusions:

a. A method is available for calculating the distribution of X-rays in uniform media with a moderate amount of labor up to fairly large depths of penetration.

b. This technique can presumably be applied with success to the broad class of transport phenomena governed by Boltzmann-type equations. It can take advantage even of rather crude theoretical predictions on the behavior of a distribution function to formulate an initial approximation that can 
thereafter be rapidly improved by straightforward numerical work.

Nevertheless, much further practical experience may be required before one can assess with some confidence the criteria for the most economical application of this technique. For example, it is not immediately apparent how far one can conveniently push the search for an improved weight function.

The present method should probably prove convenient for mapping out the distribution of various radiations within thick layers of scattering and absorbing materials. The necessary formulae for calculating the spacial moments of electron distributions have recently been developed by Lewis [11].

One can conceive of making still broader use of the methods considered in section II. There is some question as to whether the fitting of distribution functions by rapidly converging approximations should remain a problem by problem affair or whether some useful guiding principles can be formulated.

The present approach is complementary to the earlier papers $[2,3]$ which dealt primarily with the asymptotic behavior of the photon distribution and which made extensive use of the method of Laplace transforms. The work reported in those papers has now been developed into a more comprehensive treatment which will be the object of a separate report.

Continuation of this work will aim primarily in two directions:

a. To evaluate the effect of boundaries between two media and, in general, to consider the propagation through inhomogeneous barriers.

b. To include the effect of X-ray regeneration by the cascade shower mechanism, which becomes important at high energies.

\section{References}

[1] H. A. Bethe, U. Fano, and P. R. Karr, Phys. Rev. 76, 538 (1949).

[2] U. Fano, Phys. Rev. \%6, 739 (1949)

[3] U. Fano, H. Hurwitz, Jr., and L. V. Spencer, Phys. Rev. 7\%, 425 (1950)

[4] P. R. Karr and J. C. Lamkin, Phys. Rev. g6, 1843 (1949).

[5] L. V. Spencer and Fannie Jenkins, Phys. Rev. \%6, 1885 (1949).

[6] G. C. Wick, Phys. Rev. \%5, 738 (1949).

[7] R. E. Marshak, Rev. Mod. Phys. 19, 201 (1947).

[8] P. R. Karr and H. Hurwitz, Jr., in preparation.

[9] G. R. White, Phys. Rev., 80, 154 (1950).

[10] F. H. Day, NBS Cir. 507 (1951).

[11] H. W. Lewis, Phys. Rev. 78, 526 (1950).

Washington, July 20, 1950. 